\title{
Stabilization for the wave equation with Neumann boundary condition by a locally distributed damping
}

\author{
Patrick Martinez *
}

\begin{abstract}
We consider the problem of the wave equation with Neumann boundary condition damped by a locally distributed linear damping $a(x) u^{\prime}$. When the damping region $\omega:=\{x, a(x) \geq \alpha>0\}$ contains a neighborhood of the boundary of the domain, E. Zuazua proved that the energy decays exponentially to zero. Using a piecewise multiplier method introduced by K. Liu, we prove that the energy decays exponentially to zero under weaker geometrical conditions. We give explicit examples when the domain is a polyhedron, and in the case of a disc. The proof is based on the construction of multipliers adapted to the geometrical conditions.
\end{abstract}

\section{Introduction}

Let $\Omega$ be a bounded domain in $\mathbb{R}^{N}$ of class $\mathcal{C}^{1,1}$. The problem of stabilization of the wave equation with Dirichlet boundary condition damped by the use of a locally distributed feedback has been studied by several authors. Consider

$$
\left\{\begin{array}{l}
u^{\prime \prime}-\Delta u+a(x) u^{\prime}=0 \text { in } \Omega, \\
u=0 \text { on } \partial \Omega \\
u(0)=u^{0}, u^{\prime}(0)=u^{1}
\end{array}\right.
$$

Set $\alpha>0$ and define

$$
\omega:=\{x \in \Omega, a(x)>\alpha\} .
$$

As usual define the energy of the solution by

$$
E(t)=\frac{1}{2} \int_{\Omega}\left(u^{\prime 2}+|\nabla u|^{2}\right) d x
$$

It is easy to see that if $\omega=\Omega$, the energy decays exponentially to zero. E. Zuazua [12] proved that this holds true under weaker geometrical conditions: it is sufficient that $\omega$ contains a neighborhood of the part $\Gamma\left(x^{0}\right)$ of the boundary $\Gamma$ of $\Omega$ defined by

$$
\Gamma\left(x^{0}\right):=\left\{x \in \partial \Omega,\left(x-x^{0}\right) \cdot \nu(x) \geq 0\right\},
$$

*Département de Mathématiques, E. N. S. Cachan, Antenne de Bretagne, Campus de Ker Lann, 35170 Bruz, France. email: martinez@bretagne.ens-cachan.fr 
where $x^{0} \in \mathbb{R}^{N}$ and $\nu(x)$ represents the outward unit normal vector at $x \in \partial \Omega$. His proof is based on the construction of an adequate multiplier, and can be adapted to the more general case of the following semilinear wave equation :

$$
\left\{\begin{array}{l}
u^{\prime \prime}-\Delta u+a(x) u^{\prime}+f(u)=0 \text { dans } \Omega \times \mathbb{R}_{+}, \\
u=0 \text { sur } \partial \Omega \times \mathbb{R}_{+} \\
u(0)=u^{0}, u^{\prime}(0)=u^{1}
\end{array}\right.
$$

under suitable assumptions on the function $f$.

Recently, K. Liu [6] studied the observability of a class of partial differential equations. He extended the results of E. Zuazua [11] weakening the geometrical conditions on the localization of the observation region : using a piecewise multiplier method, he obtained the property of observability under very general and easily verifiable geometrical conditions. K. Liu and M. Yamamoto [7] combined this method and non harmonic analysis results developped by V. Komornik [4] to establish the observability of the system of the wave equation with Dirichlet boundary condition under the same geometrical conditions with very precise estimates of the minimal time $T_{0}$ needed for observability (that is : the system is observable in time $T$ if $T>T_{0}$.

On the other hand, C. Bardos, G. Lebeau et J. Rauch [1] proved that if $\Omega$ and $a$ are analytic, the energy of solutions of (1.1) decays uniformly exponentially to zero if and only if the damping region satisfies the "geometric optics condition". Their proof is based on microlocal analysis techniques.

Now we consider the problem of the wave equation with Neumann boundary condition damped by a locally distributed feedback :

$$
\left\{\begin{array}{l}
u^{\prime \prime}-\Delta u+a(x) u^{\prime}+q u=0 \text { in } \Omega, \\
\partial_{\nu} u=0 \text { on } \partial \Omega \\
u(0)=u^{0}, u^{\prime}(0)=u^{1}
\end{array}\right.
$$

where $q: \Omega \longrightarrow \mathbb{R}_{+}$is a nonnegative and nonzero function. We define the energy by

$$
E(t)=\frac{1}{2} \int_{\Omega}\left(u^{\prime 2}+|\nabla u|^{2}+q u^{2}\right) d x .
$$

E. Zuazua noted that the method he used in [12] to study (1.1) can be adapted to prove that the energy of the solutions of (1.5) decays exponentially if the damping region $\omega$ contains a neighborhood of the whole boundary of $\Omega$, but the question of exponential decay remained open in the case where the function $a$ is equal to zero on a part of the boundary.

The result of C. Bardos, G. Lebeau et J. Rauch [1] can still be adapted to (1.5) when the domain $\Omega$ and the function $a$ are sufficiently smooth.

In this work, by adapting the piecewise multiplier method to the difficulties related to Neumann boundary condition, we give sufficient geometrical conditions under 
which the energy of the solutions of (1.5) decays exponentially (with an explicit decay rate). Then we apply our results to some examples, constructing explict multipliers that are adapted to the given domain. This gives an answer to a question raised by E. Zuazua in [12]. In the same way, one shall study the decay rate of solutions of the semilinear wave equation with Neumann boundary condition damped by a nonlinear feedback $a(x) g\left(u^{\prime}\right)$. We applied also this method of adapted multipliers to obtain some results for the stabilization of the wave equation with a boundary feedback (see [8]).

\section{Main result}

Let $\Omega$ be a bounded open domain in $\mathbb{R}^{N}$, and $\omega$ a subdomain of $\Omega$. Let $a, q: \bar{\Omega} \longrightarrow$ $\mathbb{R}_{+}$be two nonnegative continuous functions. We assume that $x \mapsto a(x) q(x)$ is not identically equal to zero.

We consider the problem of the wave equation with Neumann boundary condition damped by a locally distributed linear feedback

$$
\begin{gathered}
u^{\prime \prime}-\Delta u+a(x) u^{\prime}+a(x) q(x) u=0 \text { in } \Omega \times \mathbb{R}_{+}, \\
\partial_{\nu} u=0 \text { on } \partial \Omega \times \mathbb{R}_{+}, \\
u(0)=u^{0}, u^{\prime}(0)=u^{1} .
\end{gathered}
$$

As usual, we define the energy by

$$
E(t)=\frac{1}{2} \int_{\Omega}\left(u^{\prime 2}+|\nabla u|^{2}+a q u^{2}\right) d x .
$$

We will use the following notations :

- $x \cdot y$ represents the euclidean scalar product of $x$ by $y$ in $\mathbb{R}^{N}$,

- if $\Omega_{j} \subset \mathbb{R}^{N}$ is a Lipschitz domain, $\nu_{j}$ represents the outward unit normal vector to the boundary $\partial \Omega_{j}$,

- if $\mathcal{O} \subset \mathbb{R}^{N}$ and $x \in \mathbb{R}^{N}, d(x, \mathcal{O}):=\inf _{y \in \mathcal{O}}|x-y|$, and $\mathcal{N}_{\varepsilon}(\mathcal{O}):=\left\{x \in \mathbb{R}^{N}\right.$ : $d(x, \mathcal{O})<\varepsilon\}$.

Geometrical conditions : assume that the following geometrical conditions on $\Omega$ and $\omega$ are satisfied :

- $\Omega$ is convex or of class $\mathcal{C}^{1,1}$. (Then there exists a unique solution $u(t)$ of the problem (2.1)-(2.3), and $u$ satisfies the following regularity

$$
u \in L^{\infty}\left(\mathbb{R}_{+}, H^{s_{0}}(\Omega)\right)
$$

with some $s_{0}>\frac{3}{2}$; this regularity is sufficient to justify the computations we will do.) 
- there exist $\varepsilon>0, \eta>0$, subdomains $\Omega_{j} \subset \Omega, 1 \leq j \leq J$, with a Lipschitz boundary $\partial \Omega_{j}$, associated functions $\phi_{j} \in \mathcal{C}^{2}\left(\overline{\Omega_{j}}\right)$ and constants $\mu_{j}$ such that

$$
\left\{\begin{array}{l}
\Delta \phi_{j}(x)-\mu_{j} \geq \eta \text { in } \Omega_{j}, \\
2 \lambda_{1, j}(x)-\Delta \phi_{j}(x)+\mu_{j} \geq \eta \text { in } \Omega_{j},
\end{array}\right.
$$

where $\lambda_{1, j}(x)$ represents the smallest eigenvalue of the symmetric matrix $D^{2} \phi_{j}(x)$.

Moreover we assume that

$$
\begin{gathered}
\Omega_{i} \cap \Omega_{j}=\emptyset \text { if } i \neq j, \\
\omega \supset \Omega \cap \mathcal{N}_{\varepsilon}\left[\cup_{j} \Gamma_{j}^{1}\left(\phi_{j}\right) \cup\left(\Omega \backslash \cup_{j} \Omega_{j}\right)\right],
\end{gathered}
$$

where

$$
\Gamma_{j}^{1}\left(\phi_{j}\right):=\left\{x \in \partial \Omega_{j}: \partial_{\nu_{j}} \phi_{j}(x) \neq 0\right\}
$$

Our main result is the following :

Theorem 1. Assume that the conditions (2.5)-(2.7) are satisfied. Assume that the function $a$ is bounded from below by a positive constant $\alpha$ on $\omega$ :

$$
a(x) \geq \alpha>0 \text { on } \omega .
$$

Then the energy decays exponentially : there exists a positive constant $\gamma$ such that

$$
E(t) \leq E(0) e^{1-\gamma t}
$$

Remark. This generalizes the result of E. Zuazua [12] who considered the case where $\omega$ contains a neighborhood of the whole boundary.

In the following, we give some examples of application of our result : first we consider the case of a polyhedron, and next we consider the (more difficult) case of a disc.

\section{Examples of convenient geometrical conditions}

The case where $\omega$ contains a neighborhood of the whole boundary (treated by E. Zuazua [12]) corresponds to the case where we have only one subdomain $\Omega_{1}$ equal to $\Omega$, and we choose

$$
\phi_{1}(x)=\frac{1}{2 N}\left|x-x^{0}\right|^{2}
$$

Indeed

$$
\forall x \in \Omega_{1}, \Delta \phi_{1}(x)=1 \text { and } \lambda_{1,1}(x)=\frac{1}{N}
$$


Therefore choosing

$$
\mu_{1}=1-\frac{1}{N} \quad \text { and } \eta=\frac{1}{N}
$$

we see that (2.5)-(2.7) are satisfied.

If the function $a$ is equal to zero on a non empty open subset $\gamma$ of the boundary $\Gamma, \omega$ is no more a neighborhood of the whole boundary. In the following, our goal is to construct $\phi$ such that its normal derivative is equal to zero on $\gamma$ :

$$
\left\{\begin{array}{l}
\Delta \phi(x)-\mu \geq \eta \text { in } \tilde{\Omega}, \\
2 \lambda_{1}(x)-\Delta \phi(x)+\mu \geq \eta \text { in } \tilde{\Omega} \\
\partial_{\nu} \phi=0 \text { on } \gamma
\end{array}\right.
$$

where $\tilde{\Omega}$ is a subdomain of $\Omega$ such that

$$
\partial \tilde{\Omega} \cap \partial \Omega=\gamma .
$$

In particular, the conditions (2.5)-(2.7) are satisfied if the following simpler ones are verified : there exist subdomains $\Omega_{j} \subset \Omega, 1 \leq j \leq J$ with Lipschitz boundary $\partial \Omega_{j}$ and functions $\phi_{j} \in \mathcal{C}^{2}\left(\overline{\Omega_{j}}\right)$ such that

$$
\left\{\begin{array}{l}
\Delta \phi_{j}(x)=1 \text { in } \Omega_{j}, \\
\lambda_{1}\left(\phi_{j}\right):=\operatorname{Inf}\left\{\lambda_{1, j}(x), x \in \Omega_{j}\right\}>0 \\
\partial_{\nu} \phi_{j}=0 \text { on } \partial \Omega_{j} \cap \partial \Omega .
\end{array}\right.
$$

Then it is sufficient to define

$$
\mu_{j}=1-\lambda_{1}\left(\phi_{j}\right) \text { on } \Omega_{j} .
$$

This remark allows us to give a first example :

\subsection{The case of a polyhedron}

Let $\Omega$ be a polyhedron in $\mathbb{R}^{N}$. We denote $\left(C_{j}\right)_{1 \leq j \leq K}$ the sides of $\Omega$ and we fix a point $x_{j}$ on each side. Let $\mathcal{O}_{j}$ be a neighborhood of $x_{j}$ in $\mathbb{R}^{N}$ such that the domain $\Omega_{j}:=\mathcal{O}_{j} \cap \Omega$ is Lipschitz and satisfies

$$
\left(\partial \Omega_{j} \cap \partial \Omega\right) \subset C_{j} .
$$

(see Fig. 1)

Then define

$$
\phi_{j}(x)=\frac{1}{2 N}\left|x-x_{j}\right|^{2} .
$$

(3.2) is satisfied thanks to the choice of $x_{j}$ :

$$
\partial_{\nu} \phi_{j}(x)=\frac{1}{N}\left(x-x_{j} \mid \nu(x)\right)=0 \text { on } \partial \Omega_{j} \cap \partial \Omega .
$$




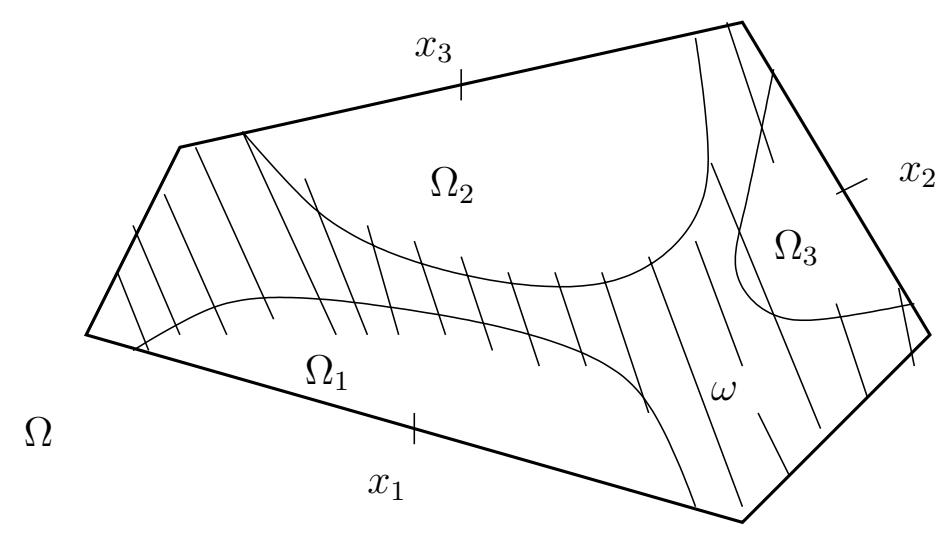

Fig. 1 : Admissible polygonal domain

( $\omega$ is represented by the hachured part.)

However, note that these special functions $\phi_{j}$ can only be used under very special geometrical conditions (that are satisfied if the domain is for example a polyhedron), but in general, we cannot find a point $x_{j}$ such that

$$
\left(x-x_{j}\right) \cdot \nu(x)=0 \text { on a part of } \partial \Omega,
$$

for example if $\Omega$ is a ball.

\subsection{The case of a disc}

If $\Omega$ is a disc in $\mathbb{R}^{2}$, it is impossible to find $x_{j} \in \mathbb{R}^{2}$ such that

$$
\left(x-x_{j}\right) \cdot \nu(x)=0 \text { on a non empty open part of } \partial \Omega .
$$

It seems also difficult to find functions $\phi_{j}$ that satisfy (3.2) (the assumption on the laplacian of $\phi_{j}$ and the one on the normal derivative of $\phi_{j}$ seem difficult to conciliate).

Let $\Omega$ be the disc $\mathcal{D}(O, R)$ centered at $O$ and of radius $R$. Since $\Omega$ is simply connected, the Riemann conformal mapping theorem (see, e.g., W. Rudin [10]) ensures us that there exists a holomorphic bijection between $\mathcal{D}(O, R)$ and the half-plane $\mathcal{P}:=\{\operatorname{Re} z<0\}$ : for example, the function

$$
z \in \mathcal{D}(O, R) \mapsto \frac{z-R}{z+R} \in \mathcal{P}
$$

is a holomorphic bijection between $\mathcal{D}(O, R)$ and $\mathcal{P}$. Define

$$
\psi: Z \in \mathcal{P} \mapsto \frac{1}{4}|Z|^{2}:
$$


the function $\psi$ satisfies

$$
\left\{\begin{array}{l}
\Delta \psi=1 \text { in } \mathcal{P} \\
\lambda_{1}(\psi)=\frac{1}{2} \\
\partial_{\nu} \psi=\frac{1}{2} z \cdot \nu(z)=0 \text { on } \partial \mathcal{P} .
\end{array}\right.
$$

This brings us to consider

$$
\phi: z \in \mathcal{D}(O, R) \mapsto\left|\frac{z-R}{z+R}\right|^{2} .
$$

We have the following

Proposition 3.1. Set $\varepsilon \in] 0,1\left[\right.$ and let $\Omega_{1}$ be the subdomain of $\Omega$ defined by

$$
\Omega_{1}:=\left\{(x, y) \in \Omega:(x-R)^{2}+y^{2}<\frac{1-\varepsilon}{256}\left((x+R)^{2}+y^{2}\right)^{4}\right\} .
$$

Then there exists $\mu_{1}$ such that $\left(\phi, \mu_{1}, \Omega_{1}\right)$ satisfies (2.5) with some $\eta>0$.

Remark. $\Omega_{1}$ is a neighborhood of the point $(R, 0)$; we will determine explicitly two subdomains of $\Omega_{1}$ (see Fig. 2).

Proof of Proposition 3.1. In order to simplify the computations, we assume that $\Omega$ is the disc $\mathcal{D}(R, R)$; we define

$$
\Phi: z \in \mathcal{D}(R, R) \mapsto\left|\frac{z-2 R}{z}\right|^{2} .
$$

Denote

$$
\operatorname{Re} z=x \text { et } \operatorname{Im} z=y \text {. }
$$

Easy computations show that

$$
\Delta \Phi(x, y)=4 \partial \bar{\partial} \Phi(z)=\frac{16 R^{2}}{|z|^{4}}
$$

and

$$
\begin{aligned}
& \frac{\partial \Phi}{\partial x}(x, y)=4 R \frac{x^{2}-2 R x-y^{2}}{|z|^{4}} \\
& \frac{\partial \Phi}{\partial y}(x, y)=8 R y \frac{x-R}{|z|^{4}} .
\end{aligned}
$$

Therefore we have the required property : since

$$
(x-R)^{2}+y^{2}=R^{2} \text { i.e. } x^{2}+y^{2}=2 R x \text { on } \partial \Omega,
$$

we have

$$
\begin{aligned}
\partial_{\nu} \Phi(x, y) & =4(x-R) \frac{x^{2}-2 R x-y^{2}}{|z|^{4}}+8 R y^{2} \frac{x-R}{|z|^{4}} \\
& =4(x-R) \frac{-2 y^{2}}{|z|^{4}}+8 R y^{2} \frac{x-R}{|z|^{4}}=0 \text { on } \partial \Omega .
\end{aligned}
$$


Next we compute the Hessian matrix of $\Phi$ :

$$
D^{2} \Phi(x, y)=\left(\begin{array}{cc}
\frac{8 R}{|z|^{4}}\left(3 x-R-\frac{4 x^{2}(x-R)}{|z|^{2}}\right) & \frac{8 R}{|z|^{4}}\left(y-\frac{4 x y(x-R)}{|z|^{2}}\right) \\
\frac{8 R}{|z|^{4}}\left(y-\frac{4 x y(x-R)}{|z|^{2}}\right) & \frac{8 R}{|z|^{4}}\left(x-R-\frac{4 y^{2}(x-R)}{|z|^{2}}\right)
\end{array}\right)
$$

Its smallest eigenvalue is

$$
\lambda_{1}(z)=\frac{8 R}{|z|^{4}}\left(R-\sqrt{(x-2 R)^{2}+y^{2}}\right) .
$$

If $(2.5)$ is satisfied on a subdomain $\Omega_{1}$, we can find $\mu_{1}$ such that

$$
\left\{\begin{array}{l}
\mu_{1} \leq \frac{16 R^{2}}{|z|^{4}}-\eta \text { dans } \Omega_{1} \\
\mu_{1} \geq \frac{16 R^{2}}{|z|^{4}}-\frac{16 R}{|z|^{4}}\left(R-\sqrt{(x-2 R)^{2}+y^{2}}\right)+\eta \text { dans } \Omega_{1} .
\end{array}\right.
$$

This is equivalent to say that

$$
\sup \left\{\frac{16 R}{|z|^{4}} \sqrt{(x-2 R)^{2}+y^{2}}, z \in \Omega_{1}\right\}<\inf \left\{\frac{16 R^{2}}{|z|^{4}}, z \in \Omega_{1}\right\} .
$$

This is clearly possible on a neighborhood of the point $(2 R, 0)$ (where the left-hand side term is equal to zero). It remains to find the largest subdomain $\Omega_{1}$ on which this property is satisfied. We note that

$$
\inf \left\{\frac{16 R^{2}}{|z|^{4}}, z \in \Omega\right\}=\frac{16 R^{2}}{(2 R)^{4}}=\frac{1}{R^{2}} .
$$

Thus this means that

$$
\sup \left\{\frac{1}{|z|^{4}} \sqrt{(x-2 R)^{2}+y^{2}}, z \in \Omega_{1}\right\}<\frac{1}{16 R^{3}} .
$$

We define $\Omega_{1}$ such that this property is satisfied.

Now consider the circle centered at $O$ and of radius $\rho<2 R$ : 


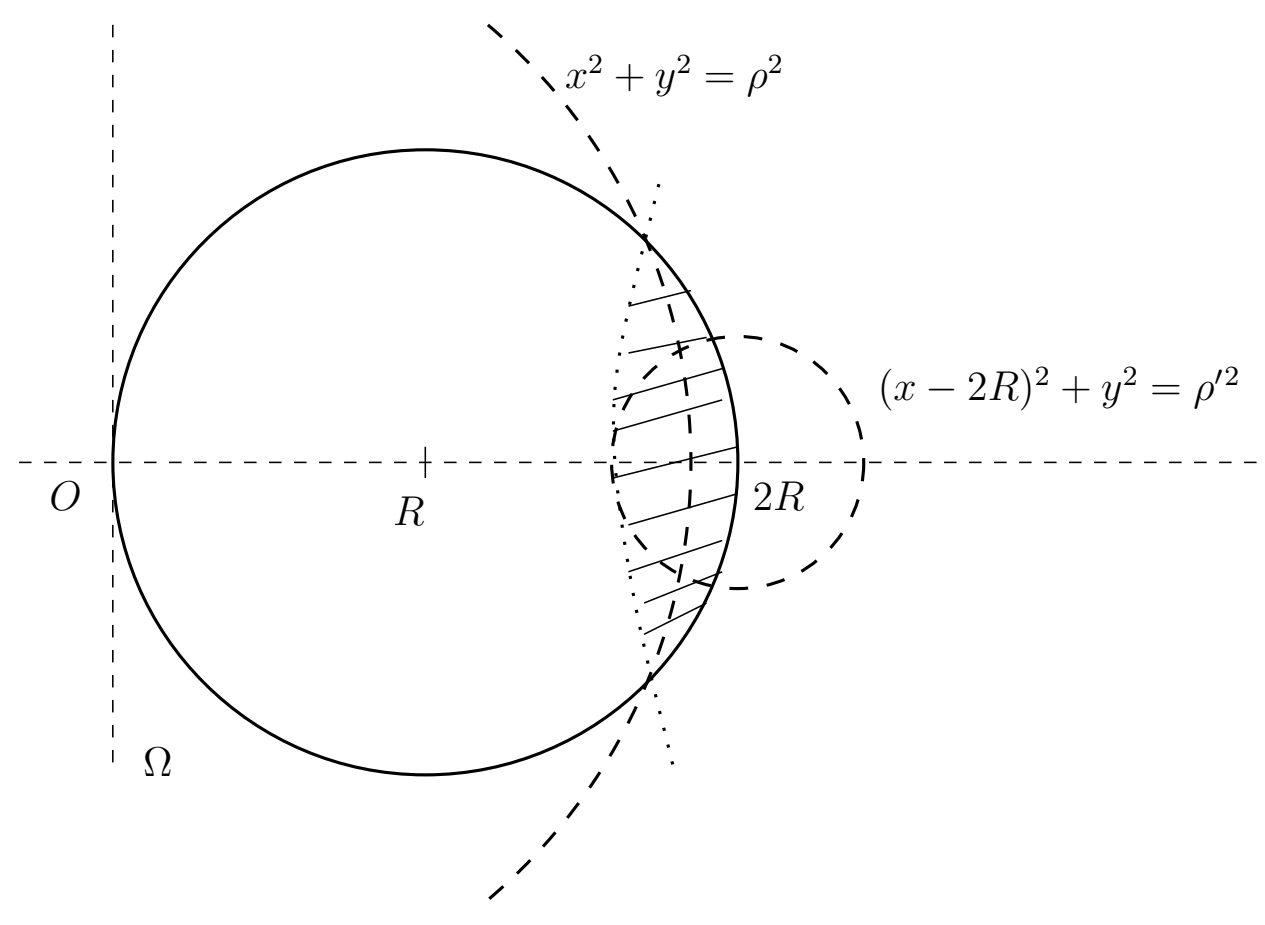

Fig. 2 : Graph of $\Omega_{1}$ (hachured part)

The circles $\mathcal{D}(R, R)$ and $\mathcal{D}(2 R, R)$ intersect themselves at the points $\left(\frac{3}{2} R, \pm \frac{\sqrt{3}}{2} R\right)$, whose modulus is $R \sqrt{3}$. Therefore, if $R \sqrt{3}<\rho<2 R$,

$$
\begin{aligned}
\sup \left\{\frac{1}{|z|^{4}} \sqrt{(x-2 R)^{2}+y^{2}},(x, y)\right. & \in \Omega \cap \mathcal{C}(O, \rho)\} \\
& =\frac{1}{\rho^{4}} \sup \left\{\sqrt{(x-2 R)^{2}+y^{2}},(x, y) \in \Omega \cap \mathcal{C}(O, \rho)\right\} .
\end{aligned}
$$

The maximum is reached at the points where the circle $\mathcal{C}(O, \rho)$ intersects $\Omega$, which are :

$$
\left\{\begin{array}{l}
(x-R)^{2}+y^{2}=r^{2} \\
x^{2}+y^{2}=\rho^{2}
\end{array}\right.
$$

this gives

$$
x=\frac{\rho^{2}}{2 R} \text { and } y= \pm \sqrt{\rho^{2}-x^{2}} .
$$

Thus

$$
\frac{1}{\rho^{4}} \sup \left\{\sqrt{(x-2 R)^{2}+y^{2}},((x, y) \in \Omega \cap \mathcal{C}(O, \rho)\}=\frac{\sqrt{4 R^{2}-\rho^{2}}}{\rho^{4}} .\right.
$$

The right-hand side term is equal to zero when $\rho=2 R$, and is nondecreasing when $\rho$ decreases. We check that if $\rho=1,86 R$, we have

$$
\frac{\sqrt{4 R^{2}-\rho^{2}}}{\rho^{4}}<\frac{1}{16 R^{3}} \text {. }
$$


Hence the zone

$$
\{z \in \Omega:|z| \geq 1,86 R\}
$$

satisfies the required geometrical conditions.

Now consider the circle $\mathcal{C}\left(2 R, \rho^{\prime}\right)$; we see that

$$
\sup \left\{\frac{1}{|z|^{4}} \sqrt{(x-2 R)^{2}+y^{2}},(x, y) \in \Omega \cap \mathcal{C}\left(2 R, \rho^{\prime}\right)\right\}=\frac{\rho^{\prime}}{\left(2 R-\rho^{\prime}\right)^{4}}
$$

and

$$
\frac{\rho^{\prime}}{\left(2 R-\rho^{\prime}\right)^{4}}<\frac{1}{16 R^{3}} \text { if } \rho^{\prime} \leq \frac{2}{5} R
$$

Hence the zone

$$
\left\{z \in \Omega:|z-2 R| \geq \frac{2}{5} R\right\}
$$

satisfies also the required geometrical conditions.

Remark. Proposition 3.1 implies that if the function $a$ is bounded from below by $\alpha>0$ on the hachured part of the following disc (Fig. 3), then the geometrical conditions required by Theorem 1 are satisfied, and the energy of weak solutions decays exponentially to zero.

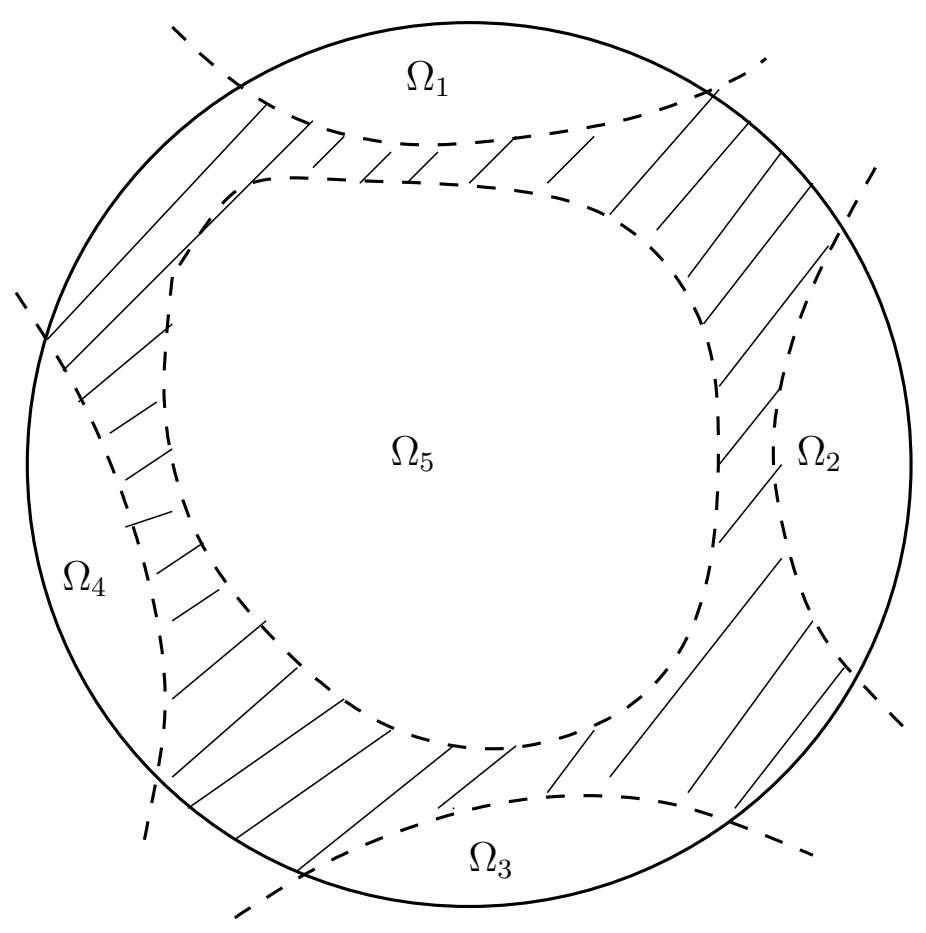

Fig. 3. Suitable geometrical conditions 


\section{Proof of Theorem 1}

First we verify that the problem (2.1)-(2.3) is dissipative :

\section{Lemma 1.}

$$
\forall 0 \leq S<T<+\infty, \quad E(S)-E(T)=\int_{S}^{T} \int_{\Omega} a(x) u^{\prime 2} d x d t .
$$

Proof of Lemma 1. We multiply (2.4) by $u^{\prime}$ and we integrate by parts on $\Omega \times[S, T]$ :

$$
\begin{aligned}
\int_{S}^{T} \int_{\Omega}-a u^{\prime 2}=\int_{S}^{T} \int_{\Omega} u^{\prime}\left(u^{\prime \prime}-\Delta\right. & u+a q u) \\
& =\left[\frac{1}{2} \int_{\Omega} u^{\prime 2}+|\nabla u|^{2}+a q u^{2}\right]_{S}^{T}=E(T)-E(S) .
\end{aligned}
$$

\subsection{The piecewise multiplier method}

Lemma 2. Let $\mathcal{O} \subset \Omega$ be a Lipschitz domain.

Let $h: \mathcal{O} \longrightarrow \mathbb{R}^{n}$ be a vector field of class $\mathcal{C}^{1}$. Set $0 \leq S<T<+\infty$. Then we have the following identity :

$$
\begin{aligned}
\int_{S}^{T} \int_{\partial \mathcal{O}} 2 \partial_{\nu} u h \cdot \nabla u & +(h \cdot \nu)\left(u^{\prime 2}-|\nabla u|^{2}\right) \\
= & {\left[\int_{\mathcal{O}} 2 u^{\prime} h \cdot \nabla u\right]_{S}^{T}+\int_{S}^{T} \int_{\mathcal{O}} 2 a\left(u^{\prime}+q u\right) h \cdot \nabla u } \\
& \quad+\int_{S}^{T} \int_{\mathcal{O}}(\operatorname{div} h)\left(u^{\prime 2}-|\nabla u|^{2}\right)+2 \sum_{i, k} \frac{\partial h_{k}}{\partial x_{i}} \frac{\partial u}{\partial x_{i}} \frac{\partial u}{\partial x_{k}}
\end{aligned}
$$

Remark. The integrations by parts leading to (4.2) are usually done if $\mathcal{O}$ is of class $\mathcal{C}^{2}$, and remain valid if $\mathcal{O}$ is only Lipschitz thanks to the results of P. Grisvard [3].

Proof of Lemma 2. This identity is given by the multiplier method : we compute

$$
\begin{gathered}
0=\int_{S}^{T} \int_{\mathcal{O}} 2 h \cdot \nabla u\left(u^{\prime \prime}-\Delta u+a\left(u^{\prime}+q u\right)\right) \\
=\left[\int_{\mathcal{O}} 2 u^{\prime} h \cdot \nabla u\right]_{S}^{T}-\int_{S}^{T} \int_{\mathcal{O}} 2 u^{\prime} h \cdot \nabla u^{\prime} \\
-\int_{S}^{T} \int_{\partial \mathcal{O}} 2 \partial_{\nu} u h \cdot \nabla u+\int_{S}^{T} \int_{\mathcal{O}} \nabla u \cdot \nabla(2 h \cdot \nabla u)+2 a\left(u^{\prime}+q u\right) h \cdot \nabla u, \\
=\left[\int_{\mathcal{O}} 2 u^{\prime} h \cdot \nabla u\right]_{S}^{T}-\int_{S}^{T} \int_{\partial \mathcal{O}} 2 \partial_{\nu} u h \cdot \nabla u+h \cdot \nu u^{\prime 2} \\
+\int_{S}^{T} \int_{\mathcal{O}}(\operatorname{div} h) u^{\prime 2}+2 \sum_{i, k}\left(\partial_{i} h_{k} \partial_{i} u \partial_{k} u+h_{k} \partial_{i} u \partial_{i, k}^{2} u\right)+2 a\left(u^{\prime}+q u\right) h \cdot \nabla u,
\end{gathered}
$$




$$
\begin{aligned}
=\left[\int_{\mathcal{O}} 2 u^{\prime} h\right. & \cdot \nabla u]_{S}^{T}-\int_{S}^{T} \int_{\partial \mathcal{O}} 2 \partial_{\nu} u h \cdot \nabla u+h \cdot \nu\left(u^{\prime 2}-|\nabla u|^{2}\right) \\
& +\int_{S}^{T} \int_{\mathcal{O}}(\operatorname{div} h)\left(u^{\prime 2}-|\nabla u|^{2}\right)+2 \sum_{i, k} \partial_{i} h_{k} \partial_{i} u \partial_{k} u+2 a\left(u^{\prime}+q u\right) h \cdot \nabla u .
\end{aligned}
$$

Hence, we get (4.2) putting the boundary integrals in the left-hand side member.

The main problem is to estimate the boundary integrals in (4.2). We will use the identity (4.2) on each subdomain $\Omega_{j}$. In the case of the wave equation with Dirichlet boundary condition, K. Liu [6] constructed a special vector field that allows us to easily estimate the boundary integrals : they are nonnegative because $u=0$ on $\partial \Omega \cap\{m \cdot \nu \leq 0\}$ and $h=0$ elsewhere (for more details see also [9]).

When we study the wave equation with Neumann boundary condition, this construction is sufficient if $\omega$ contains a neighborhood of the whole boundary of $\Omega$. But in the other cases, we have to refine this construction :

set $0<\varepsilon_{0}<\varepsilon_{1}<\varepsilon_{2}<\varepsilon$; define for $i=0$ to 2 :

$$
Q_{i}=\mathcal{N}_{\varepsilon_{i}}\left[\cup_{j} \Gamma_{j}^{1}\left(\phi_{j}\right) \cup\left(\Omega \backslash \cup_{j} \Omega_{j}\right)\right] .
$$

Since $\left(\overline{\Omega_{j}} \backslash Q_{1}\right) \cap \overline{Q_{0}}=\emptyset$, we can construct a function $\psi_{j} \in \mathcal{C}_{0}^{\infty}\left(\mathbb{R}^{n}\right)$ that satisfies

$$
\left\{\begin{array}{l}
0 \leq \psi_{j} \leq 1 \\
\psi_{j}=1 \text { on } \overline{\Omega_{j}} \backslash Q_{1} \\
\psi_{j}=0 \text { on } Q_{0}
\end{array}\right.
$$

Then we use the identity (4.2) with $\mathcal{O}:=\Omega_{j}$ et $h=h_{j}(x):=\psi_{j}(x) \nabla \phi_{j}(x)$ :

$$
\begin{array}{rl}
\int_{S}^{T} \int_{\partial \Omega_{j}} 2 \partial_{\nu_{j}} & u \psi_{j} \nabla \phi_{j} \cdot \nabla u+\left(\psi_{j} \nabla \phi_{j} \cdot \nu_{j}\right)\left(u^{\prime 2}-|\nabla u|^{2}\right) \\
= & {\left[\int_{\Omega_{j}} 2 u^{\prime} \psi_{j} \nabla \phi_{j} \cdot \nabla u\right]_{S}^{T}+\int_{S}^{T} \int_{\Omega_{j}} 2 a\left(u^{\prime}+q u\right) \psi_{j} \nabla \phi_{j} \cdot \nabla u} \\
& +\int_{S}^{T} \int_{\Omega_{j}}\left(\operatorname{div} \psi_{j} \nabla \phi_{j}\right)\left(u^{\prime 2}-|\nabla u|^{2}\right)+2 \sum_{i, k} \frac{\partial\left(\psi_{j} \nabla \phi_{j}\right)_{k}}{\partial x_{i}} \frac{\partial u}{\partial x_{i}} \frac{\partial u}{\partial x_{k}}
\end{array}
$$

First we study the boundary integrals in (4.5). Thanks to our construction, we see that

$$
\psi_{j}(x) \partial_{\nu_{j}} \phi_{j}(x)=0 \text { for all } x \in \partial \Omega_{j}
$$

Hence the second term of the boundary integrals is equal to zero. On the other hand, the first term is equal to zero on $\partial \Omega_{j} \cap \partial \Omega$ thanks to Neumann boundary condition. Since $\psi_{j}=0$ on $\partial \Omega_{j} \backslash \partial \Omega$, we see that the boundary integrals in (4.5) are equal to zero. 
Then we deduce from the definition of $\psi_{j}$ and of (4.5) that

$$
\begin{gathered}
\sum_{j}\left[\int_{\Omega_{j}} 2 u^{\prime} \psi_{j} \nabla \phi_{j} \cdot \nabla u\right]_{S}^{T}+\sum_{j} \int_{S}^{T} \int_{\Omega_{j}} 2 a\left(u^{\prime}+q u\right) \psi_{j} \nabla \phi_{j} \cdot \nabla u \\
\quad+\sum_{j} \int_{S}^{T} \int_{\Omega_{j} \backslash Q_{1}} \Delta \phi_{j}\left(u^{\prime 2}-|\nabla u|^{2}\right)+2\left(D^{2} \phi_{j} \cdot \nabla u \mid \nabla u\right) \\
=-\sum_{j} \int_{S}^{T} \int_{\Omega_{j} \cap Q_{1}}\left(\operatorname{div}\left(\psi_{j} \nabla \phi_{j}\right)\right)\left(u^{\prime 2}-|\nabla u|^{2}\right)+2 \sum_{i, k} \frac{\partial\left(\psi_{j} \nabla \phi_{j}\right)_{k}}{\partial x_{i}} \frac{\partial u}{\partial x_{i}} \frac{\partial u}{\partial x_{k}}, \\
\leq C \sum_{j} \int_{S}^{T} \int_{\Omega_{j} \cap Q_{1}} u^{\prime 2}+|\nabla u|^{2} \leq C \int_{S}^{T} \int_{\Omega \cap Q_{1}} u^{\prime 2}+|\nabla u|^{2},
\end{gathered}
$$

(Note that $\left.\cup_{j}\left(\Omega_{j} \backslash Q_{1}\right)=\Omega \backslash Q_{1}.\right)$

Set $\mu: \bar{\Omega} \longrightarrow \mathbb{R}$ a function of class $\mathcal{C}^{2}$, that remains constant on each subdomain $\Omega_{j}$ :

$$
\mu(x)=\mu_{j} \text { on } \Omega_{j}
$$

Multiplying (2.1) by $\mu u$, we get that

$$
\begin{aligned}
\int_{S}^{T} \int_{\Omega}-\mu u a\left(u^{\prime}+q u\right) & =\int_{S}^{T} \int_{\Omega} \mu u\left(u^{\prime \prime}-\Delta u\right) \\
& =\left[\int_{\Omega} \mu u u^{\prime}\right]_{S}^{T}+\int_{S}^{T} \int_{\Omega} \mu\left(-u^{\prime 2}+|\nabla u|^{2}\right)+u \nabla u \cdot \nabla \mu .
\end{aligned}
$$

Define

$$
\left\{\begin{array}{l}
h(x):=\psi_{j}(x) \nabla \phi_{j}(x) \text { if } x \in \Omega_{j}, \\
h(x):=0 \text { if } x \in \Omega \backslash \cup_{j} \Omega_{j},
\end{array}\right.
$$

and

$$
M(u):=2 h \cdot \nabla u+\mu u
$$

We add (4.7) to (4.6) and we get

$$
\begin{aligned}
& {\left[\int_{\Omega} u^{\prime} M(u)\right]_{S}^{T}+\int_{S}^{T} \int_{\Omega} a\left(u^{\prime}+q u\right) M(u) } \\
&+\sum_{j} \int_{S}^{T} \int_{\Omega_{j} \backslash Q_{1}}\left(\Delta \phi_{j}-\mu\right) u^{\prime 2}+\left(\mu-\Delta \phi_{j}\right)|\nabla u|^{2}+2\left(D^{2} \phi_{j} \cdot \nabla u \mid \nabla u\right) \\
& \leq C \int_{S}^{T} \int_{\Omega \cap Q_{1}} u^{\prime 2}+|\nabla u|^{2}+u^{2}
\end{aligned}
$$

(We used the fact that $\nabla \mu=0$ on $\Omega \backslash Q_{1}$.) 
Therefore we deduce from (2.5) that

$$
\begin{aligned}
{\left[\int_{\Omega} u^{\prime} M(u)\right]_{S}^{T}+\int_{S}^{T} \int_{\Omega} a\left(u^{\prime}+q u\right) M(u) } & \\
& +\eta \int_{S}^{T} \int_{\Omega \backslash Q_{1}} u^{\prime 2}+|\nabla u|^{2} \leq C \int_{S}^{T} \int_{\Omega \cap Q_{1}} u^{\prime 2}+|\nabla u|^{2}+u^{2}
\end{aligned}
$$

Hence

$$
\begin{aligned}
2 \eta \int_{S}^{T} E \leq-\left[\int_{\Omega} u^{\prime} M(u)\right]_{S}^{T} & -\int_{S}^{T} \int_{\Omega} a\left(u^{\prime}+q u\right) M(u) \\
& +C \int_{S}^{T} \int_{\Omega \cap Q_{1}} u^{\prime 2}+|\nabla u|^{2}+u^{2}+\eta \int_{S}^{T} \int_{\Omega} a q u^{2} .
\end{aligned}
$$

This is the key inequality to prove (2.10).

\subsection{Estimates of the right-hand side terms of (4.10)}

It is clear that

$$
\left|\int_{\Omega} u^{\prime} M(u) d x\right| \leq C_{1} E(t)
$$

On the other hand, we have the following

Lemma 3. There exists a positive constant $C$ such that, given $\delta>0$, we have

$$
\begin{aligned}
\left|\int_{S}^{T} \int_{\Omega} a\left(u^{\prime}+q u\right) M(u) d x d t\right| & \\
& \leq \frac{C}{\delta} \int_{S}^{T} \int_{\Omega} a u^{\prime 2}+a u^{2} d x d t+\delta \int_{S}^{T} E d t
\end{aligned}
$$

Proof of Lemma 3. Set $\delta>0$.

$$
\left|\int_{S}^{T} \int_{\Omega} a\left(u^{\prime}+q u\right) M(u)\right| \leq \int_{S}^{T} \int_{\Omega} \frac{\delta}{2} M(u)^{2}+\frac{c}{2 \delta}\left(a u^{\prime 2}+q a u^{2}\right) .
$$

Using the estimates (4.11) and (4.12), we deduce from (4.10) that, if $\delta$ is small enough, there exists a positive constant $C$ such that

$$
\begin{aligned}
\int_{S}^{T} E d t \leq C E(S)+ & C \int_{S}^{T} \int_{\Omega} a q u^{2}+C \int_{S}^{T} \int_{\Omega \cap Q_{1}} u^{\prime 2}+|\nabla u|^{2} d x d t \\
& \leq C E(S)+C \int_{S}^{T} \int_{\Omega} a q u^{2}+C \int_{S}^{T} \int_{\Omega \cap Q_{1}}|\nabla u|^{2} d x d t
\end{aligned}
$$

using the fact that $a(x) \geq \alpha>0$ on $\Omega \cap Q_{1}$.

It remains to estimate the last term of (4.13) : 
Lemma 4. There exists a positive constant $C$ such that, given $\delta>0$ :

$$
\int_{S}^{T} \int_{\Omega \cap Q_{1}}|\nabla u|^{2} d x \quad d t \leq C E(S)+C \int_{S}^{T} \int_{\Omega \cap Q_{2}} u^{\prime 2}+u^{2} d x d t .
$$

Proof of Lemma 4. Since $\overline{\mathbb{R}^{n} \backslash Q_{2}} \cap \overline{Q_{1}}=\emptyset$, we can construct $\xi \in \mathcal{C}_{0}^{\infty}\left(\mathbb{R}^{n}\right)$ that satisfies

$$
\left\{\begin{array}{l}
0 \leq \xi \leq 1 \\
\xi=1 \text { on } Q_{1}, \\
\xi=0 \text { on } \mathbb{R}^{n} \backslash Q_{2} .
\end{array}\right.
$$

Then we multiply (2.1) by $\xi u$ to get :

$$
\begin{aligned}
\int_{S}^{T} \int_{\Omega}-\xi u a\left(u^{\prime}+q u\right) & =\int_{S}^{T} \int_{\Omega} \xi u\left(u^{\prime \prime}-\Delta u\right) \\
= & {\left[\int_{\Omega} \xi u u^{\prime}\right]_{S}^{T}+\int_{S}^{T} \int_{\Omega}-\xi u^{\prime 2}+\nabla u \cdot \nabla(\xi u) } \\
& =\left[\int_{\Omega} \xi u u^{\prime}\right]_{S}^{T}+\int_{S}^{T} E^{\sigma} \phi^{\prime} \int_{\Omega} \xi\left(|\nabla u|^{2}-u^{\prime 2}\right)-\frac{1}{2} u^{2} \Delta \xi
\end{aligned}
$$

Hence we see that

$$
\begin{aligned}
& \int_{S}^{T} \int_{\Omega \cap Q_{1}}|\nabla u|^{2} \\
& \leq-\left[\int_{\Omega} \xi u u^{\prime}\right]_{S}^{T}+\int_{S}^{T} \int_{\Omega}-\xi u a\left(u^{\prime}+q u\right)+\xi u^{\prime 2}+\frac{1}{2} u^{2} \Delta \xi \\
& \leq C E(S)+C \int_{S}^{T} \int_{\Omega \cap Q_{2}} u^{\prime 2}+u^{2} .
\end{aligned}
$$

Finally we adapt a method introduced by F. Conrad and B. Rao [2] to estimate the last term of (4.14) :

Lemma 5. There exists a positive constant $C$ such that, given $\eta>0$ :

$$
\int_{S}^{T} \int_{\Omega} a u^{2} d x d t \leq C E(S)+\frac{C}{\eta} \int_{S}^{T} \int_{\Omega} a u^{\prime 2} d x d t+\eta \int_{S}^{T} E d t .
$$

Proof of Lemma 5. Set $t \geq 0$. We consider the solution $z$ of the following elliptic system :

$$
\begin{gathered}
-\Delta z+a q z=a u \text { in } \Omega, \\
\partial_{\nu} z=0 \text { on } \partial \Omega .
\end{gathered}
$$

We multiply (4.16) by $z$ and we get

$$
\int_{\Omega}|\nabla z|^{2}+a q z^{2}=\int_{\Omega} a u z
$$


Since $x \mapsto a(x) q(x)$ is not identically equal to zero, there exists $c>0$ such that

$$
\|z\|_{L^{2}(\Omega)}^{2} \leq c \int_{\Omega}|\nabla z|^{2}+a q z^{2} .
$$

Hence the Cauchy-Schwarz inequality gives us :

$$
\int_{\Omega} z^{2} \leq c \int_{\Omega} a u^{2}
$$

On the other hand, deriving with respect to $t$, we see that $z^{\prime}$ satisfies

$$
\begin{gathered}
-\Delta z^{\prime}+a q z^{\prime}=a u^{\prime} \text { in } \Omega, \\
\partial_{\nu} z^{\prime}=0 \text { on } \partial \Omega .
\end{gathered}
$$

Hence we deduce from this that

$$
\int_{\Omega} z^{\prime 2} \leq c \int_{\Omega} a u^{\prime 2}
$$

Next we multiply (2.1) by $z$ :

$$
\begin{aligned}
& 0=\int_{S}^{T} \int_{\Omega} z\left(u^{\prime \prime}-\Delta u+a u^{\prime}+a q u\right) \\
&=\left[\int_{\Omega} z u^{\prime}\right]_{S}^{T}-\int_{S}^{T} \int_{\Omega} z^{\prime} u^{\prime}-\int_{S}^{T} \int_{\Omega} u \Delta z+\int_{S}^{T} \int_{\Omega} a z\left(u^{\prime}+q u\right) \\
&=\left[\int_{\Omega} z u^{\prime}\right]_{S}^{T}-\int_{S}^{T} \int_{\Omega} z^{\prime} u^{\prime} \\
& \quad+\int_{S}^{T} \int_{\Omega} a u^{2}-a q u z+\int_{S}^{T} \int_{\Omega} a z\left(u^{\prime}+q u\right) .
\end{aligned}
$$

Hence

$$
\int_{S}^{T} \int_{\Omega} a u^{2}=-\left[\int_{\Omega} z u^{\prime}\right]_{S}^{T}+\int_{S}^{T} \int_{\Omega} z^{\prime} u^{\prime}-\int_{S}^{T} \int_{\Omega} a z u^{\prime}
$$

First we note that

$$
\left|\int_{\Omega} z u^{\prime}\right| \leq c\|u\|_{L^{2}(\Omega)}\left\|u^{\prime}\right\|_{L^{2}(\Omega)} \leq c E(t) .
$$

Next we see that

$$
\begin{aligned}
\left|\int_{S}^{T} \int_{\Omega} z^{\prime} u^{\prime}\right| \leq c \int_{S}^{T}\left(\int_{\Omega} a u^{\prime 2}\right)^{1 / 2} & \left(\int_{\Omega} u^{\prime 2}\right)^{1 / 2} \\
& \leq \frac{c}{\eta} \int_{S}^{T} \int_{\Omega} a u^{\prime 2}+c \eta \int_{S}^{T} \int_{\Omega} u^{\prime 2} \leq \frac{c}{\eta} E(S)+2 c \eta \int_{S}^{T} E .
\end{aligned}
$$

Finally we estimate the last term in the same way:

$$
\left|\int_{S}^{T} \int_{\Omega} a z u^{\prime}\right| \leq c \int_{S}^{T}\left(\int_{\Omega} u^{2}\right)^{1 / 2}\left(\int_{\Omega} a u^{\prime 2}\right)^{1 / 2} \leq c \eta \int_{S}^{T} E+\frac{c}{\eta} E(S) .
$$

We deduce (4.15) from (4.22) and from the last three estimates. 


\subsection{End of the proof of Theorem 1}

Using (4.14) and next (4.15), we deduce from (4.13) that

$$
\int_{S}^{T} E \leq \frac{C}{\eta} E(S)+\eta \int_{S}^{T} E .
$$

Choosing $\eta$ small enough, and letting $T$ go to infinity, we get that

$$
\int_{S}^{\infty} E \leq C E(S)
$$

Hence we conclude using a well-known Gronwall type inequality that

$$
\forall t \geq 0, E(t) \leq E(0) e^{1-t / C} .
$$

\section{References}

[1] C. Bardos, G. Lebeau and J. Rauch, Un exemple d'utilisation des notions de propagation pour le contrôle et la stabilisation des problèmes hyperboliques, proceedings of the workshop on non linear hyperbolic equations in applied sciences (edited by N. Bellono), Rendiconti del Seminario Matematico, Universita'e Politecnico Torino (Fascicolo Speciale 1988), 11-31.

[2] F. Conrad and B. Rao, Decay of solutions of wave equations in a star-shaped domain with nonlinear boundary feedback, Asymptot Anal. 7, 1993, 159-177.

[3] P. Grisvard, Elliptic problems in nonsmooth domains, Monographs and Studies in Math., 24, Pitman, Boston, 1985.

[4] V. Komornik, Exact Controllability and Stabilization. RAM : Research in Applied Mathematics. Masson, Paris; John Wiley, Ltd., Chichester, 1994.

[5] J.-L. Lions, Contrôlabilité exacte et stabilisation de systèmes distribués, Vol.1, Masson, Paris, 1988.

[6] K. Liu, Locally distributed control and damping for the conservative systems, SIAM J. Control Optim. 35 (1997), No.5, 1997.

[7] K. Liu and M. Yamamoto, On observation time duration for exact internal observability by the piecewise multiplier method, preprint of the University of Tokyo, 1997.

[8] P. Martinez, Uniform stabilization of the wave equation in almost star-shaped domains, SIAM J. Control Optim., to appear.

[9] P. Martinez, A new method to obtain decay rate estimates for dissipative systems with localized damping, to appear in the Rev. Mat. Complut. Madrid.

[10] W. Rudin, Real and complex analysis. Third edition. McGraw-Hill Book Co., New York, 1987. 
[11] E. Zuazua, Chap. 7 in J.-L. Lions [5].

[12] E. Zuazua, Exponential decay for the semi-linear wave-equation with locally distributed damping, Comm. P.D.E. 15 (1990), 205-235. 